\title{
artículos
}

\section{¿Un nuevo retrato de Velázquez? Wolfgang Guillermo, Duque de Neoburgo: Noticias sobre la significación política de su visita a la Corte Española}

\author{
Susana Fernández de Miguel \\ Investigadora vinculada a la UMA
}

RESUMEN

En el presente artículo presentamos el segundo de los pequeños retratos de los varios que pintó Velázquez a los estadistas que visitaron la Corte española durante el reinado de Felipe IV y que después pasaron a integrarse en las colecciones reales. Este retrato se corresponde con el realizado por el pintor al duque Wolfgang Wilhelm von PfalzNeuburg, durante su visita en 1624-1625. Es una obra de los primeros años madrileños de Velázquez y que permite apreciar su capacidad para captar la personalidad del retratado, que le situó desde su juventud en las más altas cotas de la pintura.

PALABRAS CLAVE: Velázquez/ Wolfgang Wilhelm duque de Neoburg/ pequeño retrato/ pintura madrileña.

An unknown Velazquez's portrait? Wolfgang Wilhelm Neoburg's Duke: Some news about politic signification of his visit to Spanish Royal Court.

ABSTRACT

In this article we present the second of small portraits of Velazquez from the several painted to the statesmen who visited the Spanish court, during the reign of Philip IV and became integrated into the royal collections. This corresponds to the portrait made by the painter to the Duke Wolfgang Wilhelm von Pfalz-Neuburg, during his visit in 1624-1625. It is a work of the early madrilenian years of Velazquez and shows his ability to capture the personality of the portrait, which stood from his youth at the top of painting.

KEY WORDS:Velázquez/ Wolfgang Wilhelm Neoburg's Duke/ little portrait/ Madrid's Paint.

Por el tamaño de esta pintura conservada en una colección particular de la provincia de Málaga, estábamos en presencia de un retrato, de pequeño formato, que posiblemente el pintor habría realizado seguramente como material de trabajo para otro de mayor empeño.Nos enfrentábamos a dos grandes desafíos, el primero, la identificación del artista y el segundo la identificación del personaje.

La reciente restauración de la obra, con una limpieza que retiró los barnices torcidos, permitió aflorar la calidad de la pintura y una mayor nitidez en los rasgos faciales del retratado1.

* FERNÁNDEZ DE MIGUEL, Susana: “¿Un nuevo retrato de Velázquez? Wolfgang Guillermo, Duque de Neoburgo: Noticias sobre la significación política de su visita a la Corte española”, en Boletín de Arte, $n^{\circ}$ 30-31 Departamento de Historia del Arte, Universidad de Málaga, 2009-2010, págs. 73-85. Fecha de recepción: Marzo de 2009.

${ }^{1}$ La restauración ha sido llevada a cabo por el taller de restauración madrileño Icono I\&R, S.C., C/ Virgen de los Peligros, $95^{\circ} \mathrm{B}, 28013$ Madrid, en el mes de Abril de 2008. 
El lienzo había sido fijado a tabla en los años de su ejecución, porque se trata de un trabajo de la época y la pintura se encontraba bien conservada, sin pérdidas de materia, salvo algunas pequeñas faltas en los márgenes de la obra, provocadas con el roce de su enmarcado, por lo que ni eran esenciales, ni afectaban a su contenido principal.

Por las características del lienzo y la preparación de la pintura, se podía constatar claramente que estábamos en presencia de un lienzo de la primera mitad del siglo XVII y perteneciente a la escuela madrileña.

El retrato estaba realizado con pincelada suelta y sin arrepentimientos, habiendo penetrado el artista perfectamente en la personalidad y psicología del personaje, plasmándolo con tal naturalismo, unido a la soltura de la pincelada y todo ello ejecutado en una unidad temporal breve por la rapidez de su ejecución, hacían concluir que el número de los pintores candidatos a la autoría quedaba reducido únicamente a los primeros pinceles del momento.

Por la época de su realización, el único candidato existente por la paleta de color utilizada, su realismo y correlativa simplicidad, su pincelada y en particular la manera con que el artista pinta las pupilas del personaje, concluimos que solo uno pudo llegar a pintarlo y éste artista no podía ser otro que el excelso sevillano y Pintor del Rey Diego de Silva y Velázquez.

Es muy posible que esta afirmación y el universal reconocimiento de esta autoría en el mundo artístico puedan tardar en llegar, pero es indudable que en algún momento este retrato tendrá que pasar a engrosar las obras del catálogo indubitado del pintor.

Sea como fuere y arriesgando en la proposición, nos reafirmamos en la catalogación que realizamos, en la esperanza que sea acogida sobre la necesidad de apertura de nuevos horizontes con los que enriquecer nuestro patrimonio artístico nacional.

Resuelta la primera de las incógnitas, nos quedaba resolver la segunda, es decir localizar la identidad del personaje.

El primer punto de referencia nos lo ofrecía la propia obra y era el Toisón que exhibía el retratado.

Felipe III a lo largo de su reinado había otorgado la distinción de la Insigne Orden del Toisón a un total de 61 caballeros, desde el núm. 286 al 346 de orden y su sucesor Felipe IV a un total de 123 caballeros, desde el número 347 al 470 de orden.

El personaje retratado podía haber recibido su nombramiento en cualquier de los dos reinados, si bien la época del retrato se corresponde con la primera mitad del seiscientos, lo que limitaba su localización al reinado de Felipe III y los primeros años del reinado de Felipe IV.

La búsqueda no fue fácil, porque no son muy abundantes los retratos de los personajes ilustres de ese momento de los que se dispone y los que existen están muy dispersos y repartidos entre instituciones públicas y colecciones privadas. 
Buscábamos un personaje que tuviera una nariz aguileña, un rostro ovalado, acentuado por una corta barba y bigote poblado ondulado hacia arriba, al uso de la época, con cabello si no rizado, sí claramente ondulado, con entradas que despejaban su frente y presidido por unos pequeños ojos, algo caídos, pero con mirada cálida y penetrante.

Ninguno de los personajes importantes en la corte madrileña respondía a estas características, por lo que nuestra búsqueda continuó con los nobles europeos que habían merecido la distinción que portaba. De todos ellos, solo uno respondía a las señales físicas que buscábamos y otros retratos de la época nos permitieron confirmar nuestras expectativas. El personaje retratado era Wolfgang Wilhelm Paltz-Neoburgo, Duque de Neoburgo, Duque de Baviera, Conde Palatino y caballero del Toisón de Oro.

Wolfgang Guillermo, como se le denominó en España, era hijo del conde palatino, el duque Felipe Luis y de Ana de Cleves y nieto, por línea materna, del duque Guillermo V el Rico y de la archiduquesa austriaca María de HabsburgoJagellón. Había nacido en Neuburg an der Donau el 4 de noviembre de 1578.

La muerte sin descendencia de su tío materno Juan Guillermo, duque de Cleves-Juliers y Berg y conde de Ravensburgo y Mark, le permitió reclamar sus derechos hereditarios sobre dichos territorios, al mismo tiempo que lo hacía el elector Juan Segismundo de Brandemburgo. La lucha entre ambos pretendientes fue cerrada con un acuerdo provisional, que aunque no favorecía a los Habsburgo, evitaba conflictos mayores.

A principios de 1614, las tensiones existentes en el Sacro Imperio y el deterioro de las relaciones entre Brandemburgo y Neoburgo estallaron en una nueva crisis en Cleves-Juliers.

Wolfgang Guillermo que se había convertido al catolicismo el 19 de Julio de 1613 por influencia de su futura esposa la hermana de Maximiliano de Baviera, temiendo una conspiración del duque de Brandemburgo para hacerse con el control de todos los territorios, decidió solicitar ayuda de Madrid, petición que fue muy bien recibida por el rey español, que le facilitó ayuda militar y en el mes de agosto de 1614 , un total de quince mil soldados del ejército de Flandes penetraron en los ducados de Cleves-Juliers para asegurar el control de Neoburgo sobre el mayor número de ciudades de los mismos.

Este movimiento militar permitió, junto con el buen hacer diplomático desplegado, que en el mes de noviembre Neoburgo firmara un tratado que le aseguraba el control de Juliers y Berg.

España se había garantizado un aliado y para asegurar este vínculo, en 1615, el rey concedió al duque de Neoburgo el nombramiento de Caballero de la Orden del Toisón de Oro².

2 AHN: ES.28079.AHN/1.1.1.255.5.1//ESTADO,7687,Exp.150: Expediente de concesión de la Orden del Toisón de Oro a Neoburgo, duque de (Wolfango Guillermo). 
Años después, en 1621, con la muerte del rey Felipe III, accedió al trono su hijo Felipe IV y con él su valido el Conde-Duque de Olivares en cuyas manos había quedado la dirección de las tareas del estado y, dentro de la política exterior, el Conde-Duque se encontró con que la tregua con Holanda se hallaba a punto de finalizar y España se veía en la necesidad de negociar una prórroga.

Aunque Bruselas era partidaria de la prórroga, el príncipe holandés Mauricio de Nassau imponía para la consecución del nuevo tratado unas condiciones que resultaban inaceptables para España, por lo que Olivares se mostraba partidario, de llegar, si era necesario, hasta la guerra, convencido de que la paz había sido aprovechada por los holandeses para hacerse con la navegación comercial entre nuestro país y el norte de Europa y que este predominio hacía peligrar la independencia española. Al mismo tiempo estimaba que, posibilitando con la paz la práctica del comercio, el adversario holandés se hacía cada vez más poderoso, como se constataba del rápido avance que habían experimentado sus compañías navales con la constitución en 1621 de la Compañía Holandesa de las Indias Occidentales, ampliando la de las Indias Orientales, que llevaba en funcionamiento desde 1602. Con este apogeo económico holandés, podían, en caso de conflicto, soportar el costo de un poderoso ejército.

Las relaciones entre los ciudadanos de ambos países tampoco eran pacíficas porque los abusos de los mercaderes holandeses en el tráfico comercial con América originaban constantes y continúas quejas de la nobleza y burguesía sevillana.

Olivares, que fundamentaba su política exterior en la conservación de todos los territorios heredados por el monarca, resolvió adoptar una estrategia defensiva en Flandes y un bloqueo de la economía holandesa, asfixiando militar y económicamente a las Provincias Unidas por tierra y por mar.

A partir de 1624, previa consulta con Bruselas, ordenó el cierre de las esclusas del Escalda, el Mosa, el Rin y el Lippe a los barcos holandeses, a la vez que procuraba convencer a los príncipes católicos alemanes de que hicieran lo mismo en sus estados.

Para preparar toda esta operación Olivares invita a Wolfang Guillermo, duque de Neoburgo a visitar Madrid. Se trata de una visita de estado en la que Olivares se proponía obtener que el duque de Neoburgo interrumpiera el tráfico fluvial del Weser, abandonando la política de neutralidad en la que se había situado, desde su acceso al poder.

A finales del mes de Septiembre de 1624, el duque de Neoburgo llegaba a España por el norte, y entraba en Madrid el 7 de Octubre de ese mismo año.

El cronista Gascón de Torquemada relata la entrada en Madrid del duque de Neoburgo en la siguiente forma ${ }^{3}$ :

3 GASCÓN DE TORQUEMADA, G.: Gaçeta y nuevas de la corte de España Gerónimo Gascón de Torquemada; continuada por su hijo Gerónimo Gascón de Tiedra ; la publica, Alfonso de Ceballos-Escalera 
"A los 7 entró en esta Corte el Duque de Neoburgo y Cleves, potentado de Alemania, de seiscientos mil ducados de renta, cuñado del Duque de Babiera. $Y$ antes que entrase se hicieron Consejos de Estado para el modo así de su recivimiento, como de la cortesía que se le havía de dar. Determinóse que el Rey y los Grandes le llamasen Dirección, como trata el Emperador a los potentados de Alemania, y él trata de la misma manera a los Grandes; pero toda la Corte le llama Altees. Es muy gran católico, y doce años a era muy grande hereje, y aora muy acepto al Emperador. Salió el Conde de Olivares a recivirle con toda la Corte, a cavallo con botas y espuelas, hasta fuera de la Puerta de Fuencarral. Vinieron por Palacio, donde se apearon, y besó la mano al Rey. Hecho esto, que era ya de noche, se bolvió a poner a cavallo , llevándole siempre el Conde a su mano derecha, y con todo el acompañamiento y más de 400 hachas, le llevaron a San Gerónimo, donde se le havía hecho el alojamiento en el quarto que Su Magestad tiene en aquél Convento. Vino el dicho Duque a la lijera, con solas ochenta personas, a tratar de sus Estados, como el Rey se los quitó a las Yslas por las fuerças, por estar entre la Frisa y Olandeses".

El duque llegaba con su séquito y servidores, entre los que se encontraban un cocinero con su ayudante y dos reposteros.

Se ordenó al Aposentador Real que diera "casa o casas bastantes que estubieren cerca de San Gerónimo para acomodar los cavalleros en las qua les pondrá veinte y seis camas, las ocho con colgaduras mas lucidas y mejores que se pudieren hallar, y las otras diez y ocho restantessean muy buenas y luzidas porque son todas para Cavalleros". Señalaron los encargados de la misión una casa en la calle del Prado y otra en la de Cantarranas y el criado del Conde de Olivares dio su aprobación. El paso siguiente fue la entrega por los ayudas del Guardajoyas de Felipe IV de la plata para el servicio del duque.

El día 9 de Octubre siguiente el duque de Neoburgo "fue a besar la mano a la Reyna Nuestra Señora ${ }^{4}$, a caballo y con grande acompañamiento". Para esta ceremonia estuvo acompañado por don Duarte de Portugal, que ofició en representación de Olivares y por 30 hachas, es decir acompañado de una escolta integrada por treinta archeros de la Guarda de Corps.

El día 14 de Octubre, con motivo de la celebración de las capitulaciones de la Marquesa de Eliche con don Ramiro Núñez de Guzmán, Marqués de Toral, tuvo lugar por la noche la celebración en Palacio de una "grandiosa máscara de cien señores a cavallo, con viçarrísimos vestidos" 5 .

La máscara o mascarada era un festejo de nobles a caballo, adornados con vestidos y libreas vistosas, que se celebraban por las noches, haciendo competicio-

y Gila, Madrid, Real Academia Matritense de Heráldica y Genealogía, 1991.

4 Ibídem., pág. 204

5 Ibídem. 
nes y carreras por parejas.

Uno de los principales asistentes fue el Duque de Neoburgo, previamente acompañado por el duque de Olivares a la Armería del Rey para que eligiera su vestimenta. Juntos se dirigieron después a Palacio para participar en la fiesta. Para el evento el duque formó pareja con el Embajador de Alemania, con quien corrió la máscara.

Ninguna otra noticia tenemos hasta el 20 de Noviembre siguiente. Ese día se organizó en la Plaza Mayor una fiesta de toros y cañas en honor del duque, a la que asistieron Sus Magestades y Alteças, que terminó con un "juego de capas y gorras, que según el cronista había sido muy lucido".

Esos días el Archiduque Carlos, que había entrado en la Península, se encontraba preparando también su entrada en la Corte.

Gascón de Torquemada relata como el día 25 de noviembre de ese mismo año se adelantaron, para recibir al Archiduque, sus sobrinos los Infantes Carlos y Fernando, encontrándole a las afueras de Madrid, en donde se unieron a su comitiva, acompañándole en la última parte de su viaje.

Llegados a Madrid, el Rey salió también a recibirle y para ello se hizo acompañar de su ilustre visitante el duque de Neoburgo. Gascón de Torquemada describió así la ceremonia:

"El Rey Nuestro Señor salió a recivir a su tío en coche, con el Duque de Neoburgo y el Conde de Olivares, hasta la cruz de piedra que está en saliendo de la Puerta que vá a Alcalá, donde todos se apearon, y acabadas las cortesías se metieron en el coche del Rey, llevando Su Magestad al Archiduque su tío a la mano hizquierda y a la proa los dos Ynfantes, y a los estrivos el Duque de Neoburgo, Conde de Ohvares, Almirante de Castilla y el Embaxador de Alemania. $Y$ al emparejar con el Monesterio de los Carmelitas Descalces, salieron los Pajes del Rey con hachas, cercaron el coche, y por calles retiradas fueron a Palacio, donde entraron por el Parque al Quarto del Rey, y en la Antecámara esperava toda la Nobleça de la Corte para acompañar a Su Magestad y Altecas al Quarto de la Reyna Nuestra Señora, en público por los Corredores, donde las tres Guardas estavan puestas en orden. Yvan delante del Rey y de su tío, los dos Ynfantes, y delante de Sus Alteças, el Duque de Neoburgo y el Conde de Olivares, con todo el acompañamiento [...]".

El duque tuvo una participación directa en todos los actos que se sucedieron a llegada, junto a los Infantes y Olivares, es decir en un lugar de honor dentro de la nobleza madrileña.

Desconocemos las actividades del duque de Neoburgo durante los dos meses siguientes, aunque consta su asistencia a las funciones teatrales y a los conciertos de música que se sucedieron en Palacio durante esos días, gustos artísticos 
que compartía con el rey español.

De todas las celebraciones organizadas en su honor, las que más fascinaron al duque fueron los conciertos palaciegos, por la calidad de la música que se hacía en el Alcázar, quedando tan interesado por ella que, para complacer a su invitado, el rey encargó al copista principal de su Capilla Real, Claudio de la Sablonara, que confeccionara un manuscrito con una compilación de los compositores españoles más famosos de su tiempo.

La compilación, que el duque llevó consigo a su regreso, incluyó un total de setenta y cinco canciones o tonos, como se denominaban entonces, con música de los compositores más famosos del momento, como eran Juan Blas de Castro, Joan Pau Pujol, Mateo Romero, Álvaro de los Ríos, Gabriel Díaz, Miguel de Arizo, entre otros, y con textos escritos también por los grandes escritores del momento, entre los que figuraba Lope de Vega.

El día 9 de Febrero de 1625 tuvo en el Pardo la celebración de una sesión del Consejo de Estado, durante el que tuvo lugar el nombramiento del duque de Neoburgo como miembro de dicho Consejo.

Tanto complacieron al duque los lugares de caza del rey que el día 18 de Febrero, obtenido el consentimiento real, inició un viaje que le llevaría a visitar los "Bosques y Casas Reales de su Magestad", entre ellos el Escorial y Aranjuez, y también Toledo con el fin de visitar su templo catedralicio. Iniciada la jornada y a los cuatro días de su partida, encontrándose en el Escorial, se vio en la necesidad de regresar a Madrid, para acudir a la convocatoria del Consejo de Estado, convocado por el Rey, para tratar la noticia llegada de Flandes relativa al ataque de los holandeses, que habían tomado una ciudad y pasado a mucho de sus ciudadanos a cuchillo.

Después de haber asistido a la sesión del Consejo, el día 26 reanudó su viaje, continuando por Aranjuez y dirigiéndose después a Toledo, en donde fue muy bien recibido, habiendo gozado el duque con la contemplación de sus monumentos.

La partida del Duque tuvo lugar el día 13 de Febrero de 1625, después de haber recibido del Rey "muchos favores y mercedes". La Reina al despedirse le hizo entrega, como obsequio, de un diamante que llamaban "Ojo de Buey", que se había tasado en treinta mil escudos de oro.

Correspondiendo a los favores recibidos el Duque dejó hecha entrega de generosos donativos a conventos, congregaciones, hospitales y mendigos y muchos obsequios, tanto en joyas, como en dinero, a los "Criados del Rey" que durante su estancia estuvieron destinados a su servicio, calculándose que en este apartado el duque había dejado unos doscientos mil ducados durante los escasos seis meses que había permanecido en la Corte.

El duque era un conocido amante del arte y contaba con una sólida y numerosa colección de pinturas, siendo el único noble alemán que había conseguido contratar en 1615 los servicios de Rubens, para la ejecución de dos cuadros con desti-

6 Ibídem., pág. 215. 


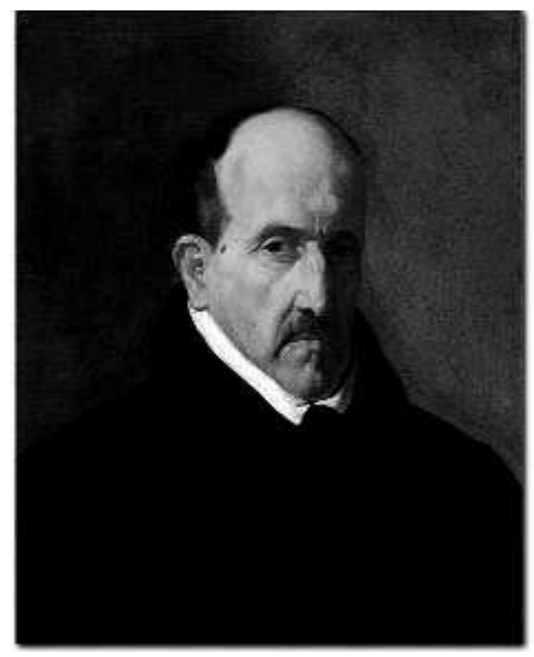

1. DIEGO VELÁZQUEZ: Retrato de don Luis de Góngora y Argote. Museum of Fine Arts. Boston.

no a la iglesia de los Jesuitas de Neoburgo y, posteriormente, los servicios de Van Dyck, quien le retrató en diversas ocasiones.

Si el Rey y su gobierno se veían en la necesidad agasajar a su noble visitante y conseguir los objetivos diplomáticos necesarios, desde el punto de vista de los intereses estratégicos de la Corona, es fácil concluir que procurarían obsequiarle en aquello que más le complaciera y ello indudablemente pasaba por el acceso al pintor que había revolucionado el panorama artístico madrileño, por su juventud y habilidad con los pinceles, a cuyo conocimiento hubiera sido imposible que se hubiera podido sustraer el Duque, cuando con Velázquez había llegado por primera vez el naturalismo psicológico a la pintura española y la sorpresa que su habilidad había provocado había ido pareja con su aceptación, sin reservas, entre mecenas y comitentes.

El Rey del estado más grande del mundo conocido, tenía a su servicio un pintor a la altura de su prestigio, Velázquez, quien constituído en vehículo del prestigio real frente a los monarcas y nobles extranjeros, era puesto a disposición de su invitado, constituyendo una de las mayores atenciones que podían concederse para tan distinguido huésped.

Políticamente la visita dio el fruto deseado y a nosotros nos dejó este bellísimo retrato, que nos ofrece la posibilidad de conocer mejor las primeras obras del Velázquez recién llegado a la Corte.

Este retrato muy bien pudiera haber pertenecido al grupo de retratos en miniatura que Juan Bautista Martínez del Mazo reseñó en el inventario que realizó del Real Alcázar en 1666 y que es seguro que tuvieron como objeto la conmemoración de las visitas de los dignatarios visitantes de la Corte, a modo de Libro de Visitas y que, en vez de firmas, eran sus rostros los que quedaban en recuerdo de su estancia 
2. ANTHONY VAN DYCK: Retrato de Wolfgang Wilhelm von Pfalz-Neuburg. Alte Pinakothek, Munich.

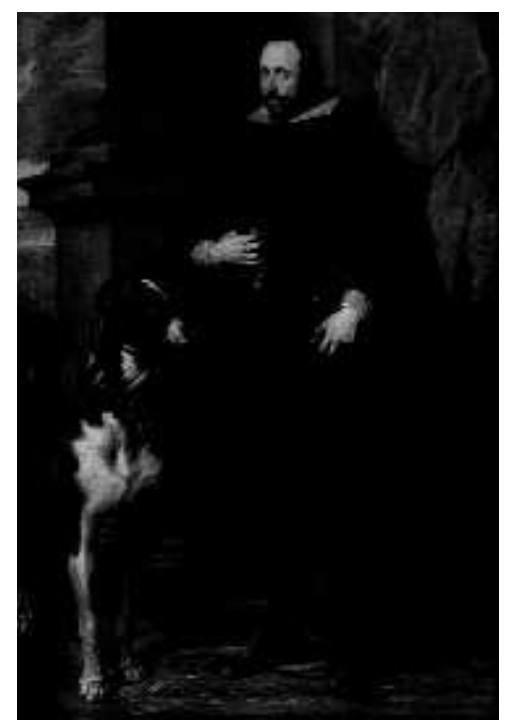

palaciega.

De esos años son muy pocas las obras que han llegado a nuestros días y las que mantienen esa datación se encuentran en mal estado o han sido sometidas a profundas restauraciones, que dificultan su apreciación en su composición original. Desconocemos si las pretensiones de Velázquez fue obtener un retrato preparatorio de otro posterior más cortesano, de cuerpo entero, o bien un pequeño retrato autónomo de recordatorio, dado que el retrato fue uno de los medios de propaganda más estimado por la nobleza a lo largo del siglo XVII. Cuando Velázquez retrata al duque de Neoburgo, éste contaba con 47 años de edad y cinco años más tarde, según la datación que se propone de la obra, fue retratado por otro de los grandes pinceles de la época Antón Van Dyck ${ }^{7}$, lo que permite asegurar la identidad del personaje en el retrato realizado por Velázquez.

Los años que median entre ambos retratos son evidentes, aunque deberíamos decir que, aparentemente, son algunos más que los que propone la datación del retrato de Van Dyck. Entre ambos retratos se aprecia claramente el paso del tiempo. En el primero el duque muestra un rostro con una piel tersa y luminosa, aún con un halo de jovialidad, atemperado por las responsabilidades que sobre el personaje recaían y que solo los primeros años de la madurez le permitían, mientras que en el segundo se percibe el desgaste que las tareas de gobierno suelen llevar con-

7 Vinculo : http://www.pinakothek.de/alte-pinakothek/kalender/kalender index en.php?haupt=ausstellungen\&inc $=$ bild\&which $=60050$ 


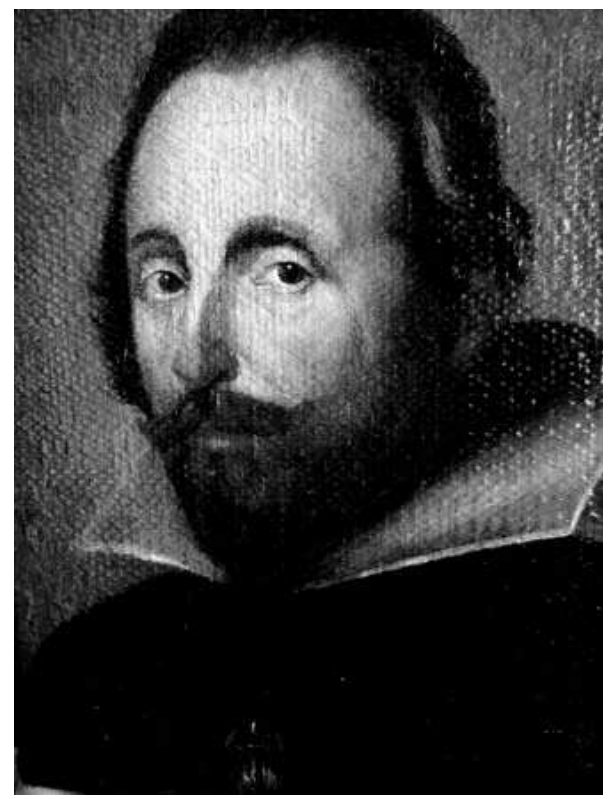

3. ¿DIEGO VELÁZQUEZ? Retrato del Duque de Neoburgo. Colección particular.

sigo. El rostro presenta ya algo de flacidez y el cabello, aunque mantiene una similar intensidad, volumen y colorido, al igual que su barba, se presentan menos poblados y más canosos. Es evidente que, desde un periodo de inicio de la madurez que se plasma en el primer retrato de Velázquez, se pasa al del inicio de la vejez que es el que recoge el retrato de Van Dyck.

El profesor Matías Díaz Padrón aseguraba en uno de sus artículos en los que comparaba la técnica de ambos artistas y haciéndose eco de lo que afirmaba Palomino cuando invitaba a los jóvenes pintores imitar a Van Dyck, cuando se tratara del halago, y a Velázquez, cuando se tratara de la verdad, que si penetrar en las almas de las criaturas fuera la categoría suprema del retrato, la superioridad de Velázquez sería indiscutible y que si lo fuera la distinción, la superioridad estaría en Van Dyck ${ }^{8}$.

La verdad es lo que debió retratar Velázquez cuando acometió este retrato, porque Van Dyck, como excelente retratista que fue, también recoge la fisonomía del duque de manera portentosa, pero no entra en la captación de la personalidad del retratado como Velázquez lo hace en el suyo, en el que a través de la mirada, que percibimos en toda su intensidad, recoge la serenidad y humanidad del personaje, como hombre culto que era, ausente de toda arrogancia y brindándose sencillamente al artista, al que supo transmitir en su cercanía la admiración que el duque sentía hacía la pintura y hacia aquéllos que tenían en sus manos la habilidad de creación de belleza.

8 DÍAZ PADRÓN, M.: "Reflexiones y precisiones del retrato de Van Dyck en la patria de Velázquez". Anales de historia del arte. № Extra 1, 2008, pág. 192. 
El espíritu de verdad que siempre inspiró a Velázquez, le llevó a modificar su técnica de plasmación de los personajes en sus lienzos, desde sus años sevillanos a sus primeros años madrileños.

Si observamos sus obras sevillanas podemos apreciar como todos los personajes no miran al espectador, ocupados en sus tareas y los menos que lo hacen mantienen una expresión de abstracción en el momento en el que el artista congela la acción. Cuando comienzan a ser demandados sus retratos, Velázquez modifica la técnica de representación. En su búsqueda de la verdad, retrata al personaje que le dirige la mirada devolviéndola y fue esta forma de representar la que revolucionó el panorama artístico madrileño.

Las obras más próximas al retrato del duque de Neoburgo, son el retrato de don Luis de Góngora y Argote $^{9}$, pintado en 1622, después de su primera visita a la Corte y el retrato del conde-Duque conservado en la Hispanic Society of America.

Aunque el retrato que presentamos está realizado casi tres años después, Velázquez mantenía aún la técnica sevillana importada, con pequeñas variantes que denotaban una adaptación al medio, comenzando lo que se ha dado en llamar etapa madrileña.

Aún así nos puede servir de comparación con ese otro primer retrato sevillano, con el que se puede establecer unos claros paralelismos en cuando a la forma de ejecución de los rasgos faciales de los personajes.

Salvando la mayor limpieza del lienzo, es patente en ambos retratos la existencia de un mismo tratamiento general de la figura y una misma manera de tratar los contornos, los ojos y la mirada del personaje. Los toques de color rosado con los que contrata el rostro son también muy similares en ambas obras.

Ningún pintor del momento supo dar tanta realidad a iris y pupilas, aquí comienzan las manchas de color que darán fama al pintor y que representan todo un universo cromático, que individualiza los retratos del artista y los distinguen de los originales de sus contemporáneos o de las copias de sus obras.

Ningún pintor hasta ese momento había conseguido tanto realismo en el retrato y esos nuevos modos son los que maravillan a los mecenas españoles y encumbran de inmediato a Velázquez.

9 Vínculo::http://www.mfa.org/collections/search art.asp?recview=true\&id=32443\&coll keywords=velazquez\&coll_accession $=\&$ coll_name $=\&$ coll_artist $=\&$ coll_place $=\&$ coll_medium $=\&$ coll_culture $=\&$ coll_classification $=\&$ coll_credit $=\&$ coll_provenance $=\&$ coll_location $=\&$ coll_has_images $=\&$ coll_on_view $=\&$ coll_sort $=0$ \&coll_so rt_order=0\&coll_view=0\&coll_package $=0 \&$ coll_start $=11$ 

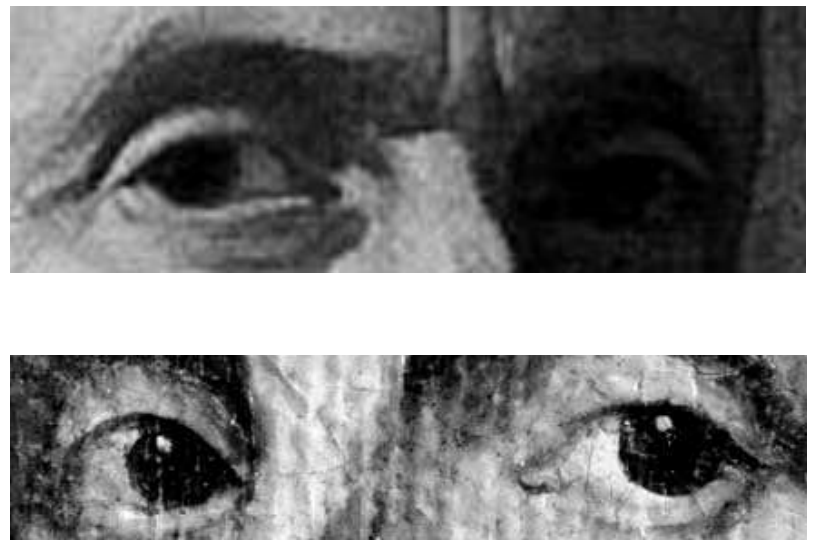

4. Retrato de

Góngora (detalle).

5. Retrato inédito del Duque de Neoburgo (detalle).

\section{EXAMEN RADIOLÓgICO DEL CUADRO}

A través de la imagen radiográfica de la obra hemos podido apreciar una excelente conservación de la obra. Apenas presenta pérdidas importantes, tan sólo alguna faltas de pequeño tamaño, que no afectan a elementos importantes de la pintura. Destaca la presencia de largas pinceladas en la parte superior del cuadro, como característica de las obras salidas de la mano de Velázquez, que le sirven para encajar el personaje dentro del lienzo. Estas pinceladas fueron utilizadas por el pintor en muchos de los cuadros de sus primeros años, como señaló Carmen Garrido Pérez en sus estudios sobre la técnica del pintor ${ }^{10}$.

En el rostro se perfilan tenuemente las líneas de enmarque de los diferentes rasgos anatómicos. Si comparamos la imagen obtenida con la del retrato del condeDuque conservado en la Hispanic Society y el de Luis de Góngora observamos una gran semejanza en las pinceladas que marcan el encajamiento de los ojos y la manera de ejecutarlos. En todas ellas coinciden los mismos puntos de luz sobre los párpados y en los breves toques de iluminación de la pupila personalísimos del pintor, que se reiteran en todas las obras.

También cabe reseñar la fina línea blanca, claramente visible en el filo de la gola, procedente de la materia pictórica que el pintor solía acumular en el extremo de las pinceladas, como recurso muy frecuente en las obras de la autoría del pintor desde su época sevillana.

Es esta obra se percibe claramente el inicio de un cambio en su técnica de la que se corresponde con su época madrileña, con fondos mucho más claros, siendo totalmente coincidentes los de este retrato con los retratos del conde-duque de Sao Paulo y el de Felipe IV del Museo del Prado.

10 GARRIDO PÉREZ, Carmen: Velázquez, Técnica y Evolución. Madrid, Museo del Prado, 1992, pág. 161. 


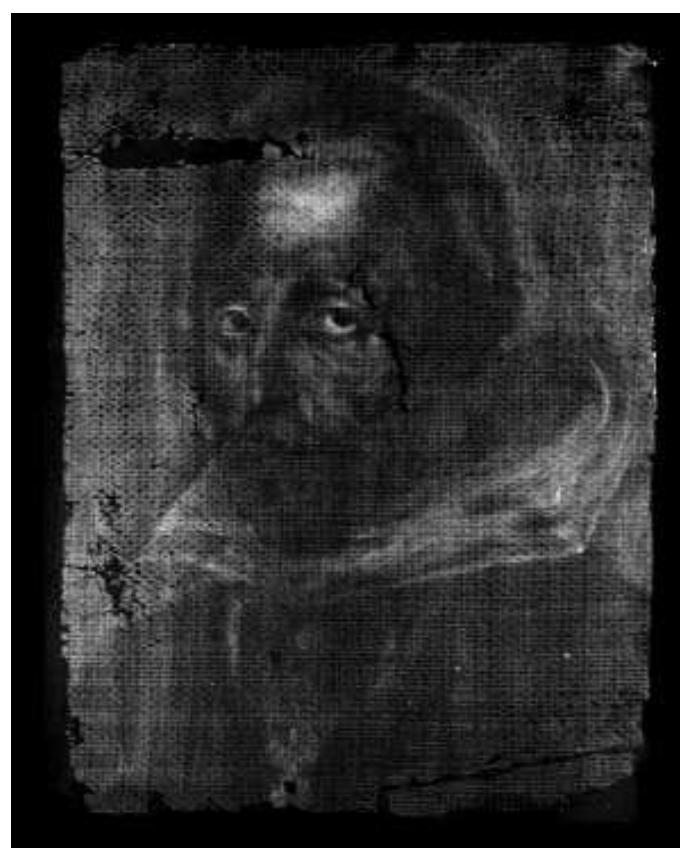

La obra presenta un menor contraste radiológico, abandonando el modelado de las figuras con abundante materia muy cargada de blanco de plomo y aligerándolos por lo que presentan una menor densidad y más rápida estructuración elaboración.

La pintura carece de correcciones o arrepentimientos, que tampoco están presentes en los rostros de otras pinturas de época coincidente con la que nos ocupa, en las que las correcciones aparecen en el cuerpo del personaje o elementos complementarios de la escena.

Las pinceladas son largas y certeras, dando vida a la figura en una realización que se muestra rápida en el tiempo, quizás en una sola sesión, evitando los largos posados a personas con múltiples e ineludibles obligaciones.

En definitiva, todos los análisis estilísticos y radiológicos abundan en mismo sentido expuesto, por lo que concluimos que nos encontramos ante una bellísima obra de Velázquez, en la que, si bien muestra la pronta madurez del pintor, también nos ofrece la excelencia de sus primeros trabajos madrileños, razones que le permitieron colocarse de inmediato a la cabeza de sus coetáneos y apunta el genio que le permitió ascender a las cotas más altas del arte de la pintura y al reconocimiento de las generaciones venideras como uno de los mayores genios de su arte. 
